\title{
Monogeneans (Dactylogyridae) parasitizing gills of Salminus hilarii from a Neotropical reservoir, Brazil
}

\author{
Monogenéticos (Dactylogyridae) parasitando brânquias de Salminus hilarii de uma represa Neotropical, Brasil
}

\author{
Heleno Brandão ${ }^{1 *}$; Fábio Hideki Yamada ${ }^{1}$; Gislayne de Melo Toledo; \\ Edmir Daniel Carvalho ${ }^{2}$; Reinaldo José da Silva ${ }^{1}$
}

\begin{abstract}
${ }^{1}$ Laboratório de Parasitologia de Animais Silvestres - LAPAS, Departamento de Parasitologia, Instituto de Biociências, UNESP - Universidade Estadual Paulista, Botucatu, SP, Brasil

${ }^{2}$ Laboratório de Biologia e Ecologia de Peixes, Departamento de Morfologia, Instituto de Biociências, UNESP - Universidade Estadual Paulista, Botucatu, SP, Brasil

$\dagger$ Edmir Daniel de Carvalho (in memory)
\end{abstract}

Received August 13, 2013

Accepted November 1, 2013

\begin{abstract}
With the aim of creating an inventory of the metazoan gill parasites of Salminus hilarii in the Taquari River, state of São Paulo, Brazil, five species of monogeneans (Anacanthorus contortus, A. bicuspidatus, Annulotrematoides parisellei, Jainus iocensis and Tereancistrum arcuatus) are reported the first time for this host. A total of 28 fish were sampled quarterly between April 2011 and January 2012, with 10 hosts in a lentic ecosystem and 18 in a lotic ecosystem. Quantitative ecological descriptors (prevalence, intensity of infestation and abundance) were calculated for the purpose to comparing the two ecosystems sampled (lentic and lotic ecosystems). However, no quantitative difference between the lentic and lotic ecosystems was observed. The present study has made available a checklist for species of the genus Anacanthorus and their hosts and geographical distribution in the Neotropical region up to the present time.
\end{abstract}

Keywords: Ectoparasite, Monogenea, freshwater fish, Taquari River.

\section{Resumo}

Com o objetivo de inventariar os metazoários parasitos de brânquias de Salminus hilarii do rio Taquari, estado de São Paulo, Brasil, cinco espécies de monogenéticos (Anacanthorus contortus, A. bicuspidatus, Annulotrematoides parisellei, Jainus iocensis, e Tereancistrum arcuatus) foram registradas pela primeira vez para o presente hospedeiro. Um total de 28 peixes foi amostrado trimestralmente entre abril/2011 e janeiro/2012, com 10 hospedeiros no ecossistema lêntico e 18 no ecossistema lótico. Os descritores ecológicos quantitativos (prevalência, intensidade de infestação e abundância) foram calculados com o objetivo de comparar os dois ecossistemas amostrados (ecossistema lêntico versus ecossistema lótico). No entanto, nenhuma diferença quantitativa desses foi verificada entre os ecossistemas lêntico e lótico. $\mathrm{O}$ presente estudo disponibiliza uma lista de espécies do gênero Anacanthorus, os hospedeiro e distribuição geográfica na região Neotropical até o presente momento.

Palavras-chave: Ectoparasito, Monogenea, peixe de água doce, rio Taquari.

\section{Introduction}

Monogeneans included in Dactylogyridae are primarily parasites of the gills of marine and freshwater fish. The class Monogenea is the most diversified group and contains the largest number of species parasitizing Neotropical fish (BOEGER; VIANNA, 2006). Specific monogeneans are common helminths parasitizing bony fish worldwide (AKOLL et al., 2012), which is a factor justifying the importance of taxonomic knowledge of species of this class.

\footnotetext{
*Corresponding author: Heleno Brandão

Departamento de Parasitologia, Instituto de Biociências, Universidade Estadual Paulista - UNESP, CEP 18618-970, Botucatu, SP, Brasil

e-mail: heleno_brandao@hotmail.com
}

Eiras et al. (2011) highlighted the increasing numbers of studies on monogeneans. However, the high number of monogenean species does not necessarily imply that this group has more species than other groups. This may simply have occurred because monogeneans have been more intensively studied than other groups.

Salminus hilarii Valenciennes, 1850, is a freshwater fish popularly known as "tabarana" in Brazil, and is considered to be a migratory species over large distances (BARBIERI et al., 2004). The geographical distribution of this species covers the main Brazilian river basins (Paraná, São Francisco, Tocantins, 
Amazon and Orinoco) (AGOSTINHO et al., 2007; GRAÇA; PAVANELLI, 2007).

The present study underscores and broadens the importance of taxonomic knowledge of species of this parasite group. This is the first study to record occurrences of monogeneans at species level, parasitizing the gills of S. hilarii. Kohn et al. (1985) identified one nematode, two digenean, and two monogenean parasites of S. hilarii, but the last ones were only identified at superfamily level (Dactylogyridae).

The Taquari River, located in the state of São Paulo, is a tributary of the Paranapanema River, which is influenced by the Jurumirim reservoir. From studies on gill parasites of $S$. hilarii in this context, occurrences of five monogenean species were reported and their quantitative parameters were compared between lentic and lotic ecosystems.

\section{Materials and Methods}

\section{Study area}

The Jurumirim dam ( $23^{\circ} 12^{\prime} 17^{\prime \prime} \mathrm{S}$ and $\left.49^{\circ} 13^{\prime} 19^{\prime \prime} \mathrm{W}\right)$ is the first of a cascade of dams on the Paranapanema River. The reservoir behind this dam is operated such that it regulates others further downstream. The dam was built in the late 1950s and operations began in 1962 (HENRY; NOGUEIRA, 1999). The Taquari River ( $23^{\circ} 15^{\prime} 12^{\prime \prime} \mathrm{S}$ and $\left.49^{\circ} 12^{\prime} 34^{\prime \prime} \mathrm{W}\right)$, located in the state of São Paulo, is the second largest tributary of the Jurumirim reservoir (HENRY et al., 1999) (Figure 1).

\section{Fish collection and laboratory procedure}

Twenty-eight specimens of $S$. hilarii from the Taquari River were collected between April 2011 and January 2012 in order to study monogenean parasites. The fish were collected using nylon monofilament gillnets with mesh sizes of 3 to $14 \mathrm{~cm}$ and with standardized effort. Nets were deployed at 5:00 pm and gathered in at 7:00 am the following day (total exposure time: $14 \mathrm{~h}$ ). Limnological parameters such as temperature $\left({ }^{\circ} \mathrm{C}\right)$, $\mathrm{pH}$ and dissolved oxygen $\left(\mathrm{mg} \mathrm{O}_{2} \cdot \mathrm{L}^{-1}\right)$ were measured with aid of a multi-parameter analyzer. Individuals were measured in terms of standard length $(L$, to the nearest $0.1 \mathrm{~mm})$ and weight (to the nearest $0.1 \mathrm{~g}$ ). The gills were frozen and subsequently removed and placed in vials containing $5 \%$ formalin solution. The parasites collected were preserved in alcohol $70 \%$. Some parasite specimens were mounted unstained in Hoyer's medium in order to study the sclerotized structures, while others were stained with Gomori's trichrome to observe internal organs (EIRAS et al., 2006).

\section{Hierarchical levels of study and statistical analysis}

In accordance with Bush et al. (1997), the following community descriptors were calculated at the infracommunity level: prevalence (\%), mean intensity of infestation and mean abundance for each parasite species. After checking the assumptions of normality (Lilliefors test), Pearson's linear correlation $(r)$ was applied to examine the relationships between parasite abundance and the limnological parameters. The Mann-Whitney $U$ test

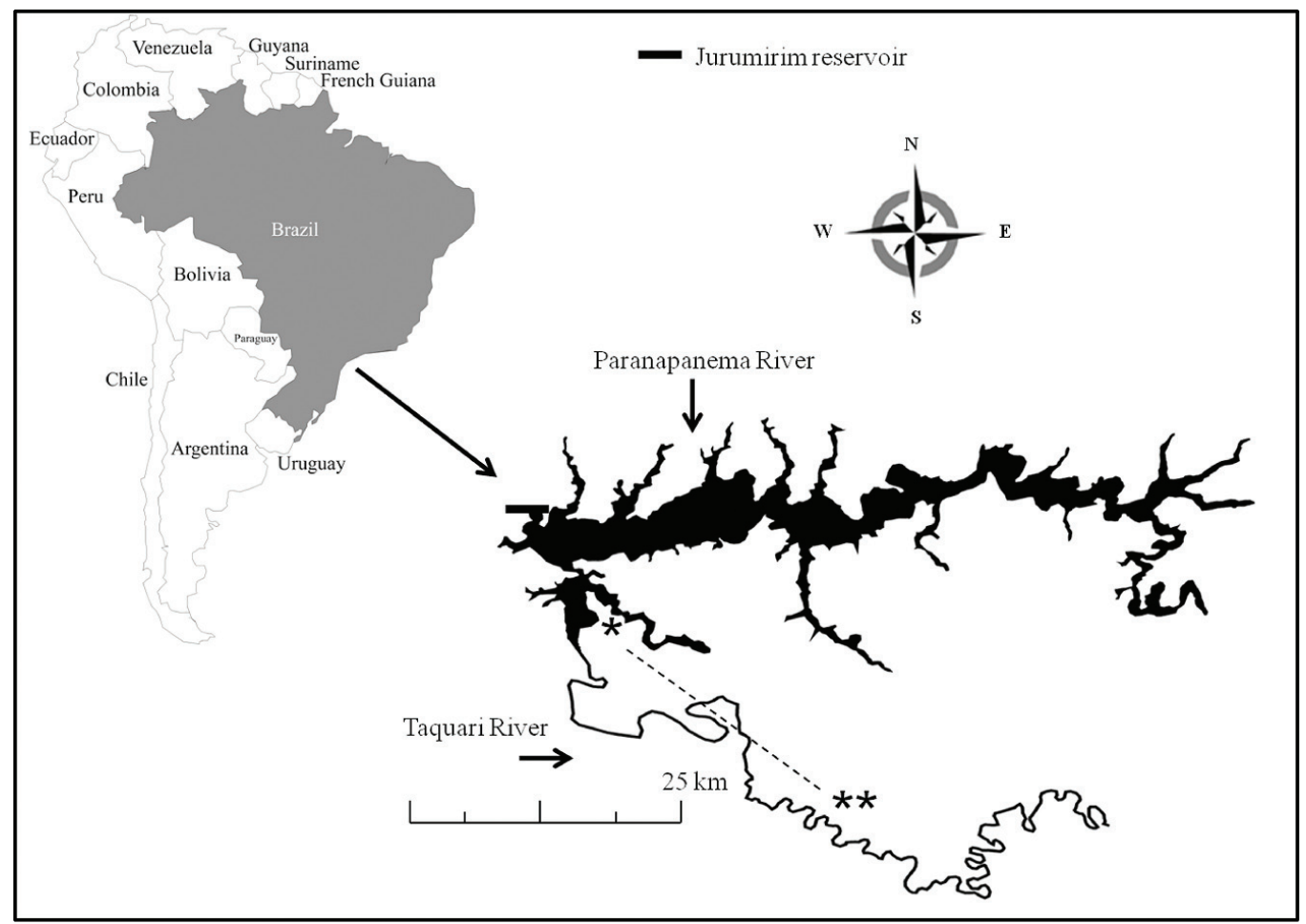

Figure 1. Jurumirim reservoir on the upper Paranapanema River, Brazil. Sampling areas: *lentic and ** lotic ecosystems in the Taquari River (arrow). Source: satellite image from Google Earth Digital Globe 
was performed to measure the effects of the lentic and lotic ecosystems on the abundance of each parasite species. Yates' corrected $X^{2}$ together with Spearman's rank correlation (rs) were also performed on pairs of co-occurring parasite species to investigate possible interspecific associations (i.e. co-operative or antagonistic relationships) (LUDWIG; REYNOLDS, 1988). The $Z$-test for proportions was performance to check differences in prevalence between infrapopulations of the lentic and lotic ecosystems.

The Berger-Parker index was applied to appraise the numerical dominance trends among parasite species (MAGURRAN, 1988). The variance-to-mean ratio of parasite abundance (dispersion index) and the discrepancy index, computed using the Quantitative Parasitology 3.0 software (RÓZSA et al., 2000), were used to detect distribution patterns of the infrapopulations (POULIN, 1993). The Shannon index (H') was calculated to compared the biological diversity of communities in the lentic and lotic ecosystems (MAGURRAN, 1988). The hierarchical levels and terminology used in this study followed Bush et al. (1997) and Poulin (2004) and the significance level used was $\mathrm{p}<0.05$.

The monogenean species were identified as described by Cohen et al. (2012) and Boeger and Vianna (2006). Voucher specimens were deposited in the Helminthological Collection of the Institute of Biosciences (CHIBB), UNESP, Botucatu, São Paulo, Brazil.

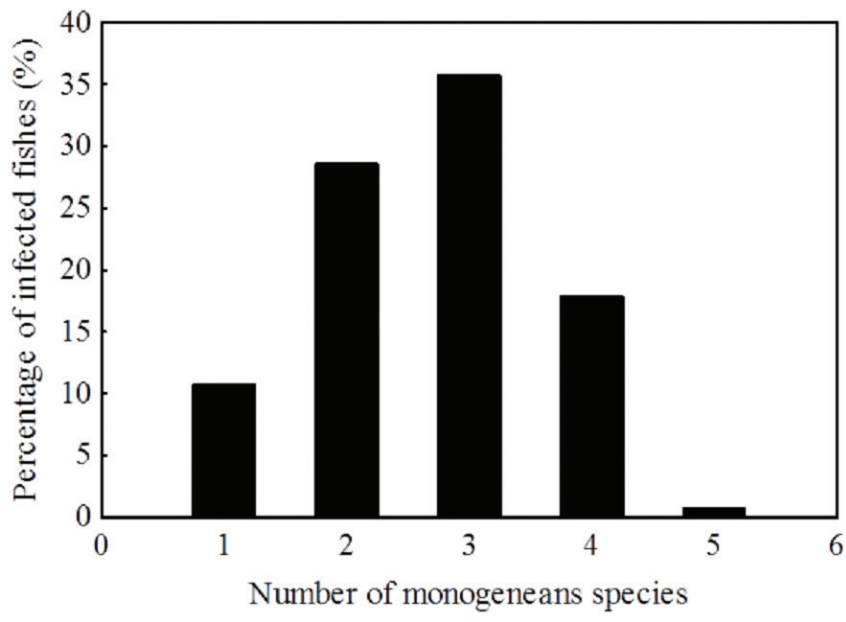

Figure 2. Richness and intensity of infection in communities of monogeneans in the gills of Salminus hilarii in the Taquari River, upper Paranapanema River, Brazil.

\section{Results}

A total of 28 fish were captured and all were parasitized by one or more monogenean species (overall prevalence $=100 \%$ ). About $35 \%$ of the fish examined were parasitized by three parasite species (Figure 2). Calculations on the absolute frequencies of the total parasites collected highlighted Anacanthorus contortus as the most important numerically (53.2\%), followed by Annulotrematoides parisellei (20.9\%), Tereancistrum arcuatus (15.1\%), Jainus iocensis (7.4\%) and $A$. bicuspidatus (3.4\%). Salminus hilarii is a new host record for all the monogeneans identified.

The component community was composed of five monogenean species totaling 417 specimens. Among these, 183 were collected in a lentic ecosystem and 234 in a lotic ecosystem, with means of $3.36 \pm 7.53$ and $2.6 \pm 4.21$, respectively. Comparing the prevalence and abundance of the component communities of the lotic and lentic ecosystems, no significant difference was observed. Fish from both ecosystems had the same magnitude of species richness and no differences in diversity $\left(\mathrm{H}_{\text {lentic }}^{\prime}=1.19\right.$ and $\mathrm{H}_{\text {lotic }}^{\prime}=1.27$; degrees of freedom $=27$; test $=0.988 ; \mathrm{p}>0.05)$.

Comparing the component communities, $A$. contortus showed high values for quantitative descriptors in both the lentic and the lotic ecosystems, except for the prevalence values. Among these, $A$. parisellei in the lentic ecosystem stood out with prevalence of $80 \%$. The parasite $A$. bicuspidatus presented the lowest prevalence and J. iocensis and T. arcuatus presented similar prevalences (Table 1).

Significant covariations of abundance were observed between pairs: $A$. contortus versus $A$. bicuspidatus, $A$. contortus versus $T$. arcuatus and $A$. parisellei versus $T$. arcuatus. Significant associations of prevalence were observed between pairs: $A$. contortus versus A. bicuspidatus and $A$. parisellei versus $A$. bicuspidatus (Table 2).

Anacanthorus contortus had the highest dominance frequency at both sites ( 0.59 and 0.48 in lentic and lotic ecosystems, respectively). All the parasites had a typically aggregated distribution pattern, and $A$. bicuspidatus was the monogenean species that showed the highest discrepancy index values (Table 3 ).

In relation to the limnological variables (Table 4), the Pearson linear correlation ( $r$ ) only revealed a statistical difference in the abundance of monogeneans in relation to $\mathrm{pH}$ : abundance versus temperature $\left(\mathrm{R}^{2}=0.81 ; \mathrm{p}=0.09\right)$; abundance versus oxygen $\left(\mathrm{R}^{2}=0.15 ; \mathrm{p}=0.61\right)$; and abundance versus $\mathrm{pH}\left(\mathrm{R}^{2}=0.89 ; \mathrm{p}=0.05\right)$. Considering the months of May to July to be the dry period and October to January to be the rainy period, greater abundance of monogeneans was observed during the rainy period (Figure 3).

Table 1. Prevalence (\%), total abundance (TA), mean intensity \pm standard error (MI \pm SE) and mean abundance (MA) of monogenean species in Salminus hilarii in the Taquari River, upper Paranapanema River, Brazil.

\begin{tabular}{|c|c|c|c|c|c|c|c|c|}
\hline \multirow{2}{*}{ Monogenean species } & \multicolumn{4}{|c|}{ Lentic ecosystem $(n=10)$} & \multicolumn{4}{|c|}{ Lotic ecosystem $(n=18)$} \\
\hline & $\%$ & TA & $\mathrm{MI} \pm \mathrm{SE}$ & MA & $\%$ & TA & $\mathrm{MI} \pm \mathrm{SE}$ & MA \\
\hline Anacanthorus contortus & 70 & 109 & $10.9 \pm 4.6$ & 7.6 & 94.4 & 114 & $6.3 \pm 1.6$ & 5.7 \\
\hline Anacanthorus bicuspidatus & 20 & 9 & $0.9 \pm 0.6$ & 0.2 & 16.7 & 5 & $0.3 \pm 0.1$ & 0.1 \\
\hline Annulotrematoides parisellei & 80 & 31 & $3.1 \pm 0.9$ & 2.5 & 77.8 & 56 & $3.1 \pm 0.8$ & 2.5 \\
\hline Jainus iocensis & 70 & 15 & $1.5 \pm 0.4$ & 1.1 & 33.3 & 16 & $0.9 \pm 0.4$ & 0.3 \\
\hline Tereancistrum arcuatus & 70 & 19 & $1.9 \pm 0.6$ & 1.3 & 38.9 & 45 & $2.5 \pm 0.7$ & 1.0 \\
\hline
\end{tabular}


Table 2. Paired associations for monogenean species in Salminus hilarii in the Taquari River, upper Paranapanema River, Brazil. Spearman rank correlation (rs). Significant values ${ }^{*} \mathrm{p} \leq 0.05$.

\begin{tabular}{lccccc}
\hline & \multicolumn{5}{c}{ Yates-corrected chi-square $\left(\chi^{2}\right)$} \\
\hline \multicolumn{1}{c}{ Monogenean species } & $\mathbf{1}$ & $\mathbf{2}$ & $\mathbf{3}$ & $\mathbf{4}$ & $\mathbf{5}$ \\
\hline (1) Anacanthorus contortus & - & $18.31^{*}$ & 0.11 & $4.88^{*}$ & 0.79 \\
(2) Anacanthorus bicuspidatus & $0.408^{*}$ & - & $18.31^{*}$ & $4.01^{*}$ & $10.62^{*}$ \\
(3) Annulotrematoides parisellei & 0.284 & 0.013 & - & $4.88^{*}$ & 0.79 \\
(4) Jainus iocensis & 0.099 & -0.007 & 0.345 & - & 1.16 \\
(5) Tereancistrum arcuatus & $0.489^{*}$ & 0.310 & $0.475^{*}$ & -0.115 & - \\
\hline
\end{tabular}

Table 3. Values of variance-to-mean ratio for parasite abundance (ID) and discrepancy index (D) among monogenean parasites of Salminus hilarii in the Taquari River, upper Paranapanema River, Brazil.

\begin{tabular}{lrr}
\hline \multicolumn{1}{c}{ Monogenean species } & ID & D \\
\hline Anacanthorus contortus & 13.256 & 0.603 \\
Anacanthorus bicuspidatus & 3.185 & 0.868 \\
Annulotrematoides parisellei & 3.441 & 0.520 \\
Jainus iocensis & 2.163 & 0.653 \\
Tereancistrum arcuatus & 3.213 & 0.577 \\
\hline
\end{tabular}

Table 4. Mean values and standard deviations of monthly limnological parameters in the Taquari River, between April 2011 and January 2012.

\begin{tabular}{cccc}
\hline Months & Temperature $\left({ }^{\circ} \mathbf{C}\right)$ & Oxygen $(\mathbf{m g} / \mathbf{L})$ & $\mathbf{p H}$ \\
\hline Apr & $18 \pm 0.9$ & $9.2 \pm 0.8$ & $5.8 \pm 0.5$ \\
July & $15.8 \pm 0.5$ & $10 \pm 0.0$ & $6.3 \pm 0.5$ \\
Oct & $23.8 \pm 0.5$ & $10 \pm 0.0$ & $7 \pm 0$ \\
Jan & $25 \pm 0.8$ & $8 \pm 1$ & $7 \pm 0$ \\
\hline
\end{tabular}

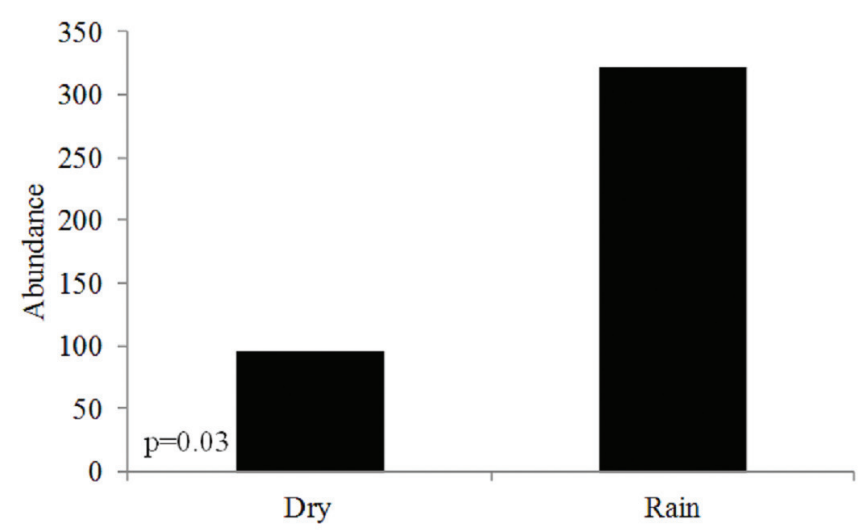

Figure 3. Comparison of the abundance of monogeneans parasitizing the gills of $S$. hilarii in the Taquari River, upper Paranapanema River, Brazil, between the dry period (April to July) and the rainy period (October to January). Significantly different values in Mann-Whitney $\mathrm{U}$ test $(\mathrm{p}<0.05)$.

\section{Discussion}

The present study reports occurrences of five monogenean species parasitizing $S$. hilarii in the Taquari River, Brazil. Individual species of Anacanthorus show varying ability to infest closely related host species. Furthermore, subgroups within Anacanthorus, based on the general morphology of the copulatory complex, appear to express high host specificity to familial groups within the Characidae. Because of these traits, species of Anacanthorus may provide valuable models for studying biogeography (KRITSKY et al., 1992). These characteristics may explain the higher abundance and mean intensity of infestations relating to $A$. contortus.

The genus Anacanthorus has large species diversity. To date, 70 species have been described, mostly from the Amazon region (Table 5). According to Van Every and Kritsky (1992), species diversity among Amazonian fish and their parasites may have resulted from lacustrine resource partitioning, similar to that proposed to explain cichlid diversity in some African lakes (LOWE-MCCONNELL, 1987) and Pliocene diversity in lake Idaho (SMITH, 1975).

So far, monogenean species of Anacanthorus have been recorded parasitizing twenty species of fish of the order Characiformes in the Neotropical region: Brycon amazonicus, Brycon melanopterus, Brycon orthotaenia, Catoprion mento, Colossoma bidens, Colossoma macropomum, Myleus rubripinnis, Mylossoma duriventris, Oreochromis mossambicus (= Tilapia mossambica), Piaractus mesopotamicus, Pristobrycon eigenmanni ( = Serrasalmus eigenmanni), Pristobrycon striolatus, Pristobrycon sp., Pygocentrus nattereri, Roeboides myersii, Salminus brasiliensis, Serrasalmus elongatus, Serrasalmus rhombeus, Serrasalmus spilopleura, Serrasalmus sp., Triportheus albus, Triportheus angulatus, Triportheus elongatus, Triportheus sp. and Salminus affinis (KRITSKY et al., 1979; KRITSKY et al., 1992; KOHN; COHEN, 1998; FISCHER et al., 2003; ANDRADE; MALTA, 2006; MONTEIRO et al., 2010; COHEN et al., 2012).

The genus Jainus has been recorded in the following hosts in South America: Brycon amazonicus, B. cephalus, B. melanopterus, Chalceus macrolepidotus, Creatochanes affinis, Leporinus copelandii, Moenkhausia sanctaefilomenae, Salminus brasiliensis and Schizodon borellii (MIZELLE et al., 1969; KRITSKY et al., 1980; KOHN; COHEN, 1998; ANDRADE et al., 2001; ANDRADE; MALTA, 2006; TAKEMOTO et al., 2009; KARLING et al., 2011; COHEN et al., 2012). Tereancistrum has been recorded in Brycon amazonicus, B. melanopterus, Leporinus fasciatus, Prochilodus reticulatus and Salminus brasiliensis (KRITSKY et al., 1980; KOHN; COHEN, 1998; ANDRADE; MALTA, 2006; COHEN et al., 2012).

During this study, we observed differences in correlations between the abundance of monogeneans and $\mathrm{pH}$, which possibly influenced the presence or absence of these organisms. However, 
Table 5. Checklist of Anacanthorus species and their hosts and geographical distribution in the Neotropical region.

\begin{tabular}{|c|c|c|c|c|}
\hline Species & Host & Family host & Locality & Reference \\
\hline \multirow[t]{3}{*}{ A. acuminatus } & Triportheus albus & Characidae & Amazon River, Brazil & Kritsky et al. (1992) \\
\hline & Triportheus angulatus & Characidae & Amazon River, Brazil & Kritsky et al. (1992) \\
\hline & Triportheus elongatus & Characidae & Amazon and Solimões Rivers, Brazil & Kritsky et al. (1992) \\
\hline \multirow[t]{2}{*}{ A. alatus } & Triportheus albus & Characidae & Amazon River, Brazil & Kritsky et al. (1992) \\
\hline & Triportheus elongatus & Characidae & Solimões River, Brazil & Kritsky et al. (1992) \\
\hline \multirow[t]{4}{*}{ A. amazonicus } & Pristobrycon striolatus & Serrasalmidae & Samaumã Pond and Uatumã River, Brazil & Van Every and Kritsky (1992) \\
\hline & Serrasalmus rhombeus & Serrasalmidae & $\begin{array}{l}\text { Agua Branca Igarape, and Pitinga, } \\
\text { Uatumá and Negro Rivers, Brazil }\end{array}$ & Van Every and Kritsky (1992) \\
\hline & Serrasalmus rhombeus & Serrasalmidae & $\begin{array}{l}\text { San Martín, Beni and Ichilo Rivers, } \\
\text { Bolivia }\end{array}$ & Córdova and Pariselle (2007) \\
\hline & Serrasalmus sp. & Serrasalmidae & $\begin{array}{l}\text { Agua Branca Igarape, and Pitinga and } \\
\text { Uatumá Rivers, Brazil }\end{array}$ & Van Every and Kritsky (1992) \\
\hline A. anacanthorus & Pygocentrus nattereri & Serrasalmidae & Amazon River, Brazil & Mizelle and Price (1965) \\
\hline A. andersoni & Triportheus angulatus & Characidae & Amazon River, Brazil & Kritsky et al. (1992) \\
\hline A. beleophallus & Pristobrycon eigenmanni & Serrasalmidae & Negro River, Brazil & Kritsky et al. (1992) \\
\hline \multirow[t]{3}{*}{ A. bellus } & Triportheus albus & Characidae & Amazon River, Brazil & Kritsky et al. (1992) \\
\hline & Triportheus elongatus & Characidae & Amazon and Solimões Rivers, Brazil & Kritsky et al. (1992) \\
\hline & Triportheus sp. & Characidae & Solimões River, Brazil & Kritsky et al. (1992) \\
\hline A. bicuspidatus & Salminus brasiliensis & Characidae & Paraná River, Brazil & Cohen et al. (2012) \\
\hline A. brasilensis & Pygocentrus nattereri & Serrasalmidae & Amazon River, Brazil & Mizelle and Price (1965) \\
\hline A. brevis & Brycon melanopterus & Characidae & Xeruiny River, Brazil & Mizelle and Kritsky (1969) \\
\hline A. brevicirrus & Brycon orthotaenia & Characidae & São Francisco River, Brazil & Monteiro et al. (2010) \\
\hline A. calophallus & Triportheus elongatus & Characidae & Solimóes River, Brazil & Kritsky et al. (1992) \\
\hline A. carinatus & Triportheus angulatus & Characidae & Amazon River, Brazil & Kritsky et al. (1992) \\
\hline A. catoprioni & Catoprion mento & Serrasalmidae & Uatumã and Amazon Rivers, Brazil & Kritsky et al. (1992) \\
\hline A. chaunophallus & Triportheus angulatus & Characidae & Amazon River, Brazil & Kritsky et al. (1992) \\
\hline \multirow[t]{2}{*}{ A. chelophorus } & Triportheus angulatus & Characidae & Amazon River, Brazil & Kritsky et al. (1992) \\
\hline & Triportheus sp. & Characidae & Solimões River, Brazil & Kritsky et al. (1992) \\
\hline A. cinctus & Pristobrycon striolatus & Serrasalmidae & Samaumã Pond and Uatumã River, Brazil & Van Every and Kritsky (1992) \\
\hline A. cladophallus & Serrasalmus spilopleura & Serrasalmidae & Solimões River, Brazil & Van Every and Kritsky (1992) \\
\hline \multirow[t]{2}{*}{ A. colombianus } & Oreochromis mossambicus & Cichlidae & Ruedasuelta Pond, Colombia & Kritsky and Thatcher (1974) \\
\hline & Salminus affinis & Characidae & Jamundi River, Colombia & Kritsky and Thatcher (1974) \\
\hline A. contortus & Salminus brasiliensis & Characidae & Paraná River, Brazil & Cohen et al. (2012) \\
\hline A. cornutus & Triportheus angulatus & Characidae & Amazon River, Brazil & Kritsky et al. (1992) \\
\hline A. crytocaulus & Pristobrycon striolatus & Serrasalmidae & $\begin{array}{l}\text { Agua Branca Igarape, Samaumã Pond and } \\
\text { Pitinga and Uatumã Rivers, Brazil }\end{array}$ & Van Every and Kritsky (1992) \\
\hline A. cuticulovaginus & Salminus affinis & Characidae & Jamundi River, Colombia & Kritsky and Thatcher (1974) \\
\hline A. daulometrus & Salminus brasiliensis & Characidae & Paraná River, Brazil & Cohen et al. (2012) \\
\hline A. dipelecinus & Roeboides myersii & Characidae & Solimóes and Negro Rivers, Brazil & Kritsky et al. (1992) \\
\hline A. douradensis & Salminus brasiliensis & Characidae & Paraná River, Brazil & Cohen et al. (2012) \\
\hline A. elegans & Brycon melanopterus & Characidae & Janauacá Lake, Brazil & Kritsky et al. (1979) \\
\hline \multirow[t]{3}{*}{ A. euryphallus } & Triportheus albus & Characidae & Amazon River, Brazil & Kritsky et al. (1992) \\
\hline & Triportheus angulatus & Characidae & Amazon River, Brazil & Kritsky et al. (1992) \\
\hline & Triportheus elongatus & Characidae & Amazon River, Brazil & Kritsky et al. (1992) \\
\hline \multirow[t]{2}{*}{ A. formosus } & Triportheus elongatus & Characidae & Amazon River, Brazil & Kritsky et al. (1992) \\
\hline & Triportheus sp. & Characidae & Solimôes River, Brazil & Kritsky et al. (1992) \\
\hline A. franciscanus & Brycon orthotaenia & Characidae & Sáo Francisco River, Brazil & Monteiro et al. (2010) \\
\hline A. furculus & Triportheus elongatus & Characidae & Solimōes River, Brazil & Kritsky et al. (1992) \\
\hline A. glyptophallus & Triportheus angulatus & Characidae & Amazon River, Brazil & Kritsky et al. (1992) \\
\hline \multirow[t]{4}{*}{ A. gravihamulatus } & Pristobrycon eigenmanni & Serrasalmidae & Uatumã River, Brazil & Van Every and Kritsky (1992) \\
\hline & Serrasalmus rhombeus & Serrasalmidae & $\begin{array}{l}\text { Agua Branca Igarape, and Pitinga and } \\
\text { Uatumá Rivers, Brazil }\end{array}$ & Van Every and Kritsky (1992) \\
\hline & Serrasalmus rhombeus & Serrasalmidae & Madre Dios River, Bolvia & Córdova and Pariselle (2007) \\
\hline & Serrasalmus sp. & Serrasalmidae & $\begin{array}{l}\text { Agua Branca Igarape, and Pitinga and } \\
\text { Uatumã Rivers, Brazil }\end{array}$ & Van Every and Kritsky (1992) \\
\hline
\end{tabular}


Table 5. Continued...

\begin{tabular}{|c|c|c|c|c|}
\hline Species & Host & Family host & Locality & Reference \\
\hline A. hoplophallus & Myleus rubripinnis & Serrasalmidae & Uatumã River, Brazil & Kritsky et al. (1992) \\
\hline \multirow[t]{6}{*}{ A. jegui } & Pristobrycon eigenmanni & Serrasalmidae & Uatumã River, Brazil & Kritsky et al. (1992) \\
\hline & Pristobrycon sp. & Serrasalmidae & Uatumã River, Brazil & Kritsky et al. (1992 \\
\hline & Serrasalmus rhombeus & Serrasalmidae & $\begin{array}{l}\text { San Martín, Beni, Madre Dios and Ichilo } \\
\text { Rivers, Bolivia }\end{array}$ & Córdova and Pariselle (2007) \\
\hline & Serrasalmus rhombeus & Serrasalmidae & $\begin{array}{l}\text { Agua Branca Igarape, and Pitinga and } \\
\text { Uatumã Rivers, Brazil }\end{array}$ & Van Every and Kritsky (1992) \\
\hline & Serrasalmus spilopleura & Serrasalmidae & Solimões River, Brazil & Kritsky et al. (1992) \\
\hline & Serrasalmus sp. & Serrasalmidae & $\begin{array}{l}\text { Agua Branca Igarape, and Pitinga, } \\
\text { Uatumã and Amazon Rivers, Brazil }\end{array}$ & Kritsky et al. (1992) \\
\hline A. kruidenieri & Brycon melanopterus & Characidae & Janauacá Lake Amazon, Brazil & Kritsky et al. (1979) \\
\hline A. lasiophallus & Pristobrycon striolatus & Serrasalmidae & $\begin{array}{l}\text { Agua Branca Igarape, Samaumã Pond and } \\
\text { Pitinga and Uatumã Rivers, Brazil }\end{array}$ & Van Every and Kritsky (1992) \\
\hline \multirow[t]{2}{*}{ A. lepyrophallus } & Serrasalmus elongatus & Serrasalmidae & Negro River, Brazil & Kritsky et al. (1992) \\
\hline & Serrasalmus sp. & Serrasalmidae & Amazon and Solimões Rivers, Brazil & Kritsky et al. (1992) \\
\hline A. lygophallus & Triportheus angulatus & Characidae & Amazon River, Brazil & Kritsky et al. (1992) \\
\hline A. maltai & Pygocentrus nattereri & Serrasalmidae & Mamoré River, Brazil & Boeger and Kritsky (1988) \\
\hline A. mastigophallus & Pristobrycon eigenmanni & Serrasalmidae & Uatumã River, Brazil & Kritsky et al. (1992) \\
\hline \multirow[t]{6}{*}{ A. mesocondylus } & Pristobrycon eigenmanni & Serrasalmidae & Uatumã and Negro Rivers, Brazil & Van Every and Kritsky (1992) \\
\hline & Pristobrycon sp. & Serrasalmidae & Uatumã River, Brazil & Van Every and Kritsky (1992) \\
\hline & Serrasalmus elongatus & Serrasalmidae & Solimóes and Negro Rivers, Brazil & Van Every and Kritsky (1992) \\
\hline & Serrasalmus rhombeus & Serrasalmidae & $\begin{array}{l}\text { Agua Branca Igarape, and Pitinga, } \\
\text { Uatumã and Negro Rivers, Brazil }\end{array}$ & Van Every and Kritsky (1992) \\
\hline & Serrasalmus spilopleura & Serrasalmidae & Solimões River, Brazil & Van Every and Kritsky (1992) \\
\hline & Serrasalmus sp. & Serrasalmidae & $\begin{array}{l}\text { Agua Branca Igarape, and Pitinga, } \\
\text { Uatumã and Solimões Rivers, Brazil }\end{array}$ & Van Every and Kritsky (1992) \\
\hline A. nanus & Triportheus angulatus & Characidae & Amazon River, Brazil & Kritsky et al. (1992) \\
\hline A. neotropicalis & Pygocentrus nattereri & Serrasalmidae & Amazon River, Brazil & Mizelle and Price (1965) \\
\hline A. palamophallus & Pristobrycon eigenmanni & Serrasalmidae & Uatumã River, Brazil & Kritsky et al. (1992) \\
\hline A. parakruidenieri & Salminus brasiliensis & Characidae & Paraná River, Brazil & Cohen et al. (2012) \\
\hline \multirow[t]{2}{*}{ A. paraspathulatus } & Mylossoma aureum & Serrasalmidae & Guandu River, Brazil & Azevedo et al. (2010) \\
\hline & Mylossoma duriventris & Serrasalmidae & Solimōes River, Brazil & Kritsky et al. (1992) \\
\hline A. pedanophallus & Myleus rubripinnis & Serrasalmidae & Uatumã River, Brazil & Kritsky et al. (1992) \\
\hline A. pelorophallus & Triportheus elongatus & Characidae & Solimões River, Brazil & Kritsky et al. (1992) \\
\hline \multirow[t]{4}{*}{ A. penilabiatus } & Colossoma macropomum & Serrasalmidae & $\begin{array}{l}\text { National Department of Public Works } \\
\text { against Drought, Brazil }\end{array}$ & Pamplona-Basilio et al. (2001) \\
\hline & Piaractus brachypomus & Serrasalmidae & $\begin{array}{l}\text { National Department of Public Works } \\
\text { against Drought, Brazil }\end{array}$ & Pamplona-Basilio et al. (2001) \\
\hline & Piaractus mesopotamicus & Serrasalmidae & Aquaculture Center (CAUNESP), Brazil & Boeger et al. (1995) \\
\hline & Piaractus mesopotamicus & Serrasalmidae & $\begin{array}{l}\text { National Department of Public Works } \\
\text { against Drought, Brazil }\end{array}$ & Pamplona-Basilio et al. (2001) \\
\hline A. periphallus & Serrasalmus sp. & Serrasalmidae & Amazon River, Brazil & Kritsky et al. (1992) \\
\hline A. pithophallus & Triportheus angulatus & Characidae & Amazon River, Brazil & Kritsky et al. (1992) \\
\hline \multirow[t]{3}{*}{ A. prodigiosus } & Serrasalmus elongatus & Serrasalmidae & Negro River, Brazil & Van Every and Kritsky (1992) \\
\hline & Serrasalmus rhombeus & Serrasalmidae & $\begin{array}{l}\text { Agua Branca Igarape, and Pitinga, } \\
\text { Uatumã and Negro Rivers, Brazil }\end{array}$ & Van Every and Kritsky (1992) \\
\hline & Serrasalmus sp. & Serrasalmidae & $\begin{array}{l}\text { Agua Branca Igarape, and Pitinga, } \\
\text { Uatumã and Solimóes Rivers, Brazil }\end{array}$ & Van Every and Kritsky (1992) \\
\hline \multirow[t]{3}{*}{ A. quinqueramus } & Triportheus albus & Characidae & Amazon River, Brazil & Kritsky et al. (1992) \\
\hline & Triportheus elongatus & Characidae & Amazon River, Brazil & Kritsky et al. (1992) \\
\hline & Triportheus sp. & Characidae & Solimóes Rives, Brazil & Kritsky et al. (1992) \\
\hline A. ramosissimus & Serrasalmus elongatus & Serrasalmidae & Solimóes River, Brazil & Van Every and Kritsky (1992) \\
\hline \multirow[t]{2}{*}{ A. ramulosus } & Triportheus albus & Characidae & Amazon River, Brazil & Kritsky et al. (1992) \\
\hline & Triportheus elongatus & Characidae & Amazon River, Brazil & Kritsky et al. (1992) \\
\hline
\end{tabular}


Table 5. Continued...

\begin{tabular}{|c|c|c|c|c|}
\hline Species & Host & Family host & Locality & Reference \\
\hline A. reginae & Pygocentrus nattereri & Serrasalmidae & $\begin{array}{l}\text { Solimóes River, Brazil, and Amazon River, } \\
\text { Loreto, Peru }\end{array}$ & $\begin{array}{l}\text { Boeger and Kritsky (1988), Ian- } \\
\text { nacone and Luque (1993) }\end{array}$ \\
\hline \multirow[t]{2}{*}{ A. rondonensis } & Pygocentrus nattereri & Serrasalmidae & Mamoré River, Brazil & Boeger and Kritsky (1988) \\
\hline & Serrasalmus rhombeus & Serrasalmidae & Madre Dios River, Bolivia & Córdova and Pariselle (2007) \\
\hline A. scapanus & Serrasalmus spilopleura & Serrasalmidae & Solimões River, Brazil & Van Every and Kritsky (1992) \\
\hline \multirow[t]{5}{*}{ A. sciponophallus } & Serrasalmus elongatus & Serrasalmidae & Solimões and Negro Rivers, Brazil & Van Every and Kritsky (1992) \\
\hline & Serrasalmus rhombeus & Serrasalmidae & $\begin{array}{l}\text { Agua Branca Igarape, and Pitinga, } \\
\text { Uatumã and Negro Rivers, Brazil }\end{array}$ & Van Every and Kritsky (1992) \\
\hline & Serrasalmus rhombeus & Serrasalmidae & $\begin{array}{l}\text { San Martin, Beni, Madre Dios and Ichilo } \\
\text { Rivers, Bolivia }\end{array}$ & Córdova and Pariselle (2007) \\
\hline & Serrasalmus spilopleura & Serrasalmidae & Solimões River, Brazil & Van Every and Kritsky (1992) \\
\hline & Serrasalmus sp. & Serrasalmidae & $\begin{array}{l}\text { Solimóes, Uatumá and Negro Rivers, } \\
\text { Brazil }\end{array}$ & Van Every and Kritsky (1992) \\
\hline \multirow[t]{4}{*}{ A. serrasalmi } & Pristobrycon sp. & Serrasalmidae & Uatumã River, Brazil & Van Every and Kritsky (1992) \\
\hline & Serrasalmus elongatus & Serrasalmidae & Solimóes and Negro Rivers, Brazil & Van Every and Kritsky (1992) \\
\hline & Serrasalmus rhombeus & Serrasalmidae & $\begin{array}{l}\text { Agua Branca Igarape, and Pitinga, } \\
\text { Uatumã and Negro Rivers, Brazil }\end{array}$ & Van Every and Kritsky (1992) \\
\hline & Serrasalmus sp. & Serrasalmidae & $\begin{array}{l}\text { Agua Branca Igarape, and Pitinga, } \\
\text { Uatumã and Negro Rivers, Brazil }\end{array}$ & Van Every and Kritsky (1992) \\
\hline \multirow[t]{3}{*}{ A. spatulatus } & Colossoma bidens & Serrasalmidae & Janauacá Lake Amazon, Brazil & Kritsky et al. (1979) \\
\hline & Colossoma macropomum & Serrasalmidae & Janauacá Lake Amazon, Brazil & Kritsky et al. (1979) \\
\hline & Colossoma macropomum & Serrasalmidae & Delta Amacuro and Portuguesa, Venezuela & $\begin{array}{l}\text { Aragort et al. (2002), Cen- } \\
\text { teno et al. (2004) }\end{array}$ \\
\hline A. spinatus & Myleus rubripinnis & Serrasalmidae & Uatumá River, Brazil & Kritsky et al. (1992) \\
\hline \multirow[t]{2}{*}{ A. spiralocirrus } & Brycon amazonicus & Characidae & Amazon River, Brazil & Andrade and Malta (2006) \\
\hline & Brycon melanopterus & Characidae & Janauacá Lake Amazon, Brazil & Kritsky et al. (1979) \\
\hline \multirow[t]{3}{*}{ A. stachophallus } & Pygocentrus nattereri & Serrasalmidae & Amazon and Solimóes Rivers, Brazil & Kritsky et al. (1992) \\
\hline & Pygocentrus nattereri & Serrasalmidae & Solimões River, Brazil & Boeger and Kritsky (1988) \\
\hline & Pygocentrus nattereri & Serrasalmidae & Amazon River, Loreto, Peru & Iannacone and Luque (1993) \\
\hline A. stagmophallus & Myleus rubripinnis & Serrasalmidae & Uatumã River, Brazil & Kritsky et al. (1992) \\
\hline A. strongylophallus & Triportheus elongatus & Characidae & Solimóes River, Brazil & Kritsky et al. (1992) \\
\hline A. thatcheri & Pygocentrus nattereri & Serrasalmidae & Solimões River, Brazil & Boeger and Kritsky (1988) \\
\hline \multirow[t]{2}{*}{ A. tricornis } & Triportheus angulatus & Characidae & Amazon River, Brazil & Kritsky et al. (1992) \\
\hline & Triportheus elongatus & Characidae & Solimões River, Brazil & Kritsky et al. (1992) \\
\hline \multirow[t]{2}{*}{ A. xaniophallus } & Pristobrycon eigenmanni & Serrasalmidae & Uatumã River, Brazil & Kritsky et al. (1992) \\
\hline & Pristobrycon sp. & Serrasalmidae & Uatumã and Negro Rivers, Brazil & Kritsky et al. (1992) \\
\hline
\end{tabular}

studies on the physiology of the host are needed in order to confirm the degree of influence of the $\mathrm{pH}$ of these organisms.

Regarding parasite dispersion, in most cases, the parasites are almost universally aggregated between their hosts (KRASNOV; POULIN, 2010). That is to say, most of the hosts have few if any parasites, while a small number of hosts are infected with many parasites (POULIN, 1993). This pattern is expected in most animals in nature, as observed regarding monogeneans parasitizing the gills of $S$. hilarii in the present study, except for $A$. pariselle $i$ in the lentic gradient. This type of distribution is possibly related to variations in the characteristics of the environment or the behavior of living beings that try to group (NERING; ZUBEN, 2010).

According Thomas et al. (2005), considerable progress has been made in understanding the functional value of parasites in ecosystems. Numerous theoretical and empirical studies have shown that parasites, in spite of their small size, are biologically and ecologically important in ecosystems. The influence of monogeneans on fish behavior in nature is difficult to measure, but we considered them within the life history of the host in order to gain better understanding of both organisms within their ecological niches.

Knowledge of the geographical and systematic distribution of monogenean species is also of considerable importance, since these organisms can be used as tools to aid in understanding, conserving and preserving aquatic ecosystems.

The present study provides the first record of occurrences of the monogeneans $A$. contortus, A. bicuspidatus, $A$. parisellei, $J$. iocensis and T. arcuatus parasitizing the gills of the host $S$. hilarii in the Taquari River in the state of São Paulo, Brazil. This study contributes towards the knowledge of occurrences of these species of parasites in fish of the family Characidae and their geographical distribution, as well as listing the host species and geographical distribution of the genus Anacanthorus in South America. 


\section{Acknowledgements}

The authors thank Fundaçáo de Amparo à Pesquisa do Estado de São Paulo (FAPESP process: 2011/22603-3) for the scholarship granted to the first author and Coordenaçáo de Aperfeiçoamento de Pessoal de Nível Superior (CAPES process: AUX-PE-PNPD 3005/2010). They are grateful to the staff of the Fish Biology and Ecology Laboratory of the Institute of Biosciences, Botucatu, and Universidade Estadual Paulista (UNESP) for the structure provided for developing this work.

\section{References}

Agostinho AA, Gomes LC, Pelicice FM. Ecologia e Manejo de Recursos Pesqueiros em Reservatórios do Brasil. Maringá: Eduem; 2007.

Akoll P, Fioravanti ML, Konecny R, Schiemer F. Infection dynamics of Cichlidogyrus tilapiae and C. sclerosus (Monogenea, Ancyrocephalinae) in Nile tilapia (Oreochromis niloticus L.) from Uganda. J Helminthol 2012; 86(3): 302-310. PMid:21791155. http://dx.doi. org/10.1017/S0022149X11000411

Andrade SMS, Malta JCO. Parasite fauna monitoring of matrinxâ Brycon amazonicus (Spix \& Agassiz, 1829) raised in an intensive husbandry system a stream channel in the state of Amazonas, Brazil. Braz J Biol 2006; 66(4): 1123-1132. http://dx.doi.org/10.1590/S151969842006000600020

Andrade SMS, Malta JC, Ferraz E. Fauna parasitológica de alevinos de Matrinchã, Brycon cephalus (Günther, 1869) coletados nos rios Negro e Solimóes, na Amazônia Central. Acta Amaz 2001; 31(2): 263-273.

Aragort W, Morales G, Leon E, Pino LA, Guillén A, Silva M. Patologías asociadas a monogeneos branquiales en cachama bajo cultivo. Vet Trop 2002; 27(2): 75-85.

Azevedo RK, Abdallah VD, Luque JL. Acanthocephala, Annelida, Arthropoda, Myxozoa, Nematoda and Platyhelminthes parasites of fishes from the Guandu river, Rio de Janeiro, Brazil. Check List 2010; 6(4): 659-667.

Barbieri G, Salles AF, Cestarolli MA, Teixeira-Filho AR. Estratégias reprodutivas do dourado, Salminus maxillosus, e do curimbatá, Prochilodus lineatus no rio Mogi Guaçu, Estado de São Paulo, com ênfase nos parâmetros matemáticos da dinâmica populacional. Acta Sci Biol Sci 2004; 26(2): 169-174. http://dx.doi.org/10.4025/actascibiolsci. v26i2.1631

Boeger WA, Kritsky DC. Neotropical Monogenea. 12. Dactylogyridae from Serrasalmus nattereri (Cypriniformes, Serrasalmidae) and aspects of their morphologic variation and distribution in the Brazilian Amazon. Proc Helminthol Soc Wash 1988; 55(2): 188-213.

Boeger WA, Vianna RT. Monogenoidea. In: Thatcher VE. Amazon Fish Parasites. Sofia: Pensoft Publishers. 2006. p. 42-116.

Boeger WA, Husak WS, Martins ML. Neotropical monogenoidea. 25. Anacanthorus penilabiatus n. sp. (Dactylogyridae, Anacanthorinae) from Piaractus mesopotamicus (Osteichthyes, Serrasalmidae), cultivated in the State of São Paulo, Brazil. Mem Inst Oswaldo Cruz 1995; 90(6): 699-701. http://dx.doi.org/10.1590/S0074-02761995000600008

Bush AO, Lafferty KD, Lotz JM, Shostak AW. Parasitology meets ecology on its own terms: Margolis et al. revisited. J Parasitol 1997; 83(4): 575583. PMid:9267395. http://dx.doi.org/10.2307/3284227
Centeno L, Silva-Acuña A, Silva-Acuña R, Pérez JL. Fauna Ectoparasitaria Asociada a Colossoma macropomum y al Híbrido de C. macropomum x Piaractus brachypomus, Cultivados en el Estado Delta Amacuro, Venezuela. Bioagro 2004; 16(2): 121-126.

Cohen SC, Kohn A, Boeger WA. Neotropical Monogenoidea. 57. Nine new species of Dactylogyridae (Monogenoidea) from the gill of Salminus brasiliensis (Characidae, Characiformes) from the Paraná River, State of Paraná, Brazil. Zootaxa 2012; 3049: 57-68.

Córdova L, Pariselle A. Monogenoidea en Serrasalmus rhombeus (Linnaeus, 1766) de la Cuenca Amazónica Boliviana. Rev Peru Biol 2007; 14(1): 11-16.

Eiras JC, Takemoto RM, Pavanelli GC, Adriano EA. About the biodiversity of parasites of freshwater fish from Brazil. Bull Eur Assoc Fish Pathol 2011; 31(4): 161-168.

Eiras JC, Takemoto RM, Pavanelli GC. Métodos de Estudo e Técnicas Laboratoriais em Parasitologia de Peixes. Maringá: Eduem; 2006.

Fischer C, Malta JCO, Varella AMB. A Fauna de Parasitas do Tambaqui, Colossoma macropomum (CUVIER, 1818) (Characiformes: Characidae) do Médio Rio Solimóes, Estado do Amazonas (AM) e do Baixo Rio Amazonas, Estado do Pará (PA), e seu Potencial como Indicadores Biológicos. Acta Amaz 2003; 33(4): 651-662.

Graça WJ, Pavanelli CS. Peixes da planície de inundação do alto rio Paraná e áreas adjacentes. Maringá: Eduem; 2007.

Henry R, Nogueira MG. A Represa de Jurumirim (São Paulo): Primeira síntese sobre o conhecimento limnológico e uma proposta preliminar de manejo ambiental. In: Henry R. Ecologia de reservatórios: estrutura, função e aspectos sociais. Botucatu: FUNDIBIO, FAPESP; 1999. p. 651-686

Henry R, Santos AAN, Camargo YR. Transporte de sólidos suspensos, $\mathrm{N}$ e P total pelos Rios Paranapanema e Taquari e uma avaliaçáo de sua exportação na represa de Jurumirim. In: Henry R. Ecologia de reservatórios: estrutura, função e aspectos sociais. Botucatu: FUNDIBIO, FAPESP; 1999. p. 687-710.

Iannacone JA, Luque JL. New records of helminths parasitic on Peruvian Amazonian fishes (Osteichthyes). Rev Biol Trop 1993; 41(2): 303-305.

Karling LC, Bellay S, Takemoto RM, Pavanelli GC. A new species of Jainus (Monogenea), gill parasite of Schizodon borellii (Characiformes, Anostomidae) from the upper Paraná river floodplain, Brazil. Acta Sci Biol Sci 2011:33(2): 227-231. http://dx.doi.org/10.4025/actascibiolsci. v33i2.6168

Kohn A, Cohen SC. South American Monogenea - list of species, hosts and geographical distribution. Int J Parasitol 1998; 28(10): 1517-1554. http://dx.doi.org/10.1016/S0020-7519(98)00083-6

Kohn A, Fernandes BMM, Macedo B, Abramson B. Helminths parasites of Freshwater fishes from Pirassununga, SP, Brazil. Mem Inst Oswaldo Cruz 1985; 80(3): 327-336. http://dx.doi.org/10.1590/S007402761985000300009

Krasnov BR, Poulin R. Ecological properties of a parasite: species specific stability and geographical variation. In: Morand S, Krasnov BR. The biogeography of host-parasite interactions. New York: Oxford University Press Inc.; 2010. p. 99-114.

Kritsky DC, Thatcher V. Monogenetic trematodes (Monopisthocotylea: Dactylogyridae) from freshwater fishes of Colombia, South America. J Helminthol 1974; 48(1): 59-66. http://dx.doi.org/10.1017/ S0022149X00022604 
Kritsky DC, Thatcher VE, Kayton RJ. Neotropical Monogenoidea 2. The Anacanthorinae Price, 1967, with the proposal of four new species of Anacanthorus Mizelle \& Price, 1965, from Amazonian fishes. Acta Amaz 1979; 9(2): 355-361.

Kristky DC, Thatcher VE, Kayton RJ. Neotropical Monogenoidea. 3. Five new species from South America with the proposal of Tereancistrum gen. n. and Trinibaculum gen. n. (Dactylogyridae: Ancyrocephalinae). Acta Amaz 1980; 10(2): 411-417.

Kritsky DC, Boeger WA, Van Every LR. Neotropical Monogenoidea. 17. Anacanthorus Mizelle and Price, 1965 (Dactylogyridae, Anacanthorinae) from Characoid Fishes of the Central amazon. J Helminthol Soc Wash 1992; 59(1): 25-51.

Lowe-McConnell RH. Ecological Studies in Tropical Fish Communities. Cambridge: Cambridge University Press; 1987. http://dx.doi. org/10.1017/CBO9780511721892

Ludwig JA, Reynolds JF. Statistical Ecology: a Primer on Methods and Computing. New York: Wiley-Interscience Publications; 1988.

Magurran AE. Ecological diversity and its measurement. New Jersey: Princeton University Press; 1988. http://dx.doi.org/10.1007/978-94015-7358-0

Mizelle JD, Kritsky DC. Studies on monogenetic trematodes. XL. New species from marine and freshwater fishes. Am Midl Nat 1969; 82(2): 417-428. http://dx.doi.org/10.2307/2423787

Mizelle JD, Price CE. Studies on Monogenetic Trematodes. XXVIII. Gill Parasites of the Piranha with Proposal of Anacanthorus gen. n. J Parasitol 1965; 51(1): 30-36. PMid:14259477. http://dx.doi. org/10.2307/3275640

Mizelle JD, Kritsky DC, Crane JW. Studies on monogenetic trematodes. XXXVIII. Ancyrocephalinae from South America with the proposal of Jainus gen. n. Am Midl Nat 1969; 80(1): 186-198. http://dx.doi. org/10.2307/2423609

Monteiro CM, Kritsky DC, Brasil-Sato MC. Neotropical Monogenoidea. 56. New species of Anacanthorus (Dactylogyridae) from the gills of matrinchã, Brycon orthotaenia (Characiformes: Characidae), in the Rio Sáo Francisco, Brazil. Folia Parasitol 2010; 57(3): 164-168.

Nering MB, Zuben CJV. Métodos Quantitativos em Parasitologia. Jaboticabal: Funep; 2010.

Pamplona-Basilio MC, Kohn A, Feitosa VA. New Host Records and Description of the Egg of Anacanthorus penilabiatus (Monogenea, Dactylogyridae). Mem Inst Oswaldo Cruz 2001; 96(5): 667668. PMid:11500767. http://dx.doi.org/10.1590/S007402762001000500014

Poulin R. The disparity between observed and uniform distributions - A new look at parasite aggregation. Int J Parasitol 1993; 23(7): 937-944. http://dx.doi.org/10.1016/0020-7519(93)90060-C

Poulin R. Macroecological patterns of species richness in parasite assemblages. Basic Appl Ecol 2004; 5(5): 423-434. http://dx.doi. org/10.1016/j.baae.2004.08.003

Rózsa L, Reiczigel J, Majoros G. Quantifying parasites in samples of hosts. J Parasitol 2000; 86(2): 228-232. PMid:10780537.

Smith GR. Fishes of the Pliocene Glenns Ferry Formation, Southwest Idaho. Claude W Hibbard Memorial 1975; 5(14): 1-68.

Takemoto RM, Pavanelli GC, Lizama MAP, Lacerda ACF, Yamada FH, Moreira LHA, et al. Diversity of parasites of fish from the Upper Paraná River floodplain, Brazil. Braz J Biol 2009; 69(2 Supll): 691-705.

Thomas F, Bonsall MB, Dobson AP. Parasitism, biodiversity, and conservation. In: Thomas F, Renaud F, Guégan J. Parasitism and Ecosystems. New York: Oxford University Press; 2005. p. 124-139. http:// dx.doi.org/10.1093/acprof:oso/9780198529873.003.0009

Van Every LR, Kritsky DC. Neotropical Monogenoidea. 18. Anacanthorus Mizelle and Price, 1965 (Dactylogyridae, Anacanthorinae) of Piranha (Characoidea, Serrasalmidae) from the Central Amazon, their Phylogeny, and aspects of Host-Parasite Coevolution.J Helminthol Soc Washington 1992; 59(1): 52-75. 